\title{
Effects of Adding Midazolam and Sufentanil to Intrathecal Bupivacaine on Analgesia Quality and Postoperative Complications in Elective Cesarean Section
}

\author{
Abolfazl Abdollahpour ${ }^{1}$; Raheleh Azadi ${ }^{1}$; Razieh Bandari ${ }^{2}$; Majid Mirmohammadkhani ${ }^{3,}$ \\ ${ }_{1}^{1}$ Department of Anesthesiology, Kowsar Hospital, Semnan University of Medical Sciences, Semnan, Iran \\ ${ }_{3}^{2}$ Department of Nursing, School of Rehabilitation, University of Social Welfare and Rehabilitation Sciences, Tehran, Iran \\ ${ }_{3}^{3}$ Research Center for Social Determinants of Health, Department of Commiunity Medicine, School of Medicine, Semnan University of Medical Sciences, Semnan, Iran \\ ${ }^{*}$ Corresponding author: Majid Mirmohammadkhani, Research Center for Social Determinants of Health, Department of Commiunity Medicine, School of Medicine, Semnan \\ University of Medical Sciences, Semnan, Iran. Tel: +98-2333654162, E-mail: majid mirmohammadkhani@yahoo.com \\ Received: September 12, 2014; Revised: November 24, 2014; Accepted: January 31, 2015
}

\begin{abstract}
Background: Intrathecal adjutants can be used for regional anesthesia (RA) in cesarean section to improve its quality in terms of time and complications. Some previous studies focused on the effects of adding sufentanil and/or midazolam to bupivacaine and compared each with using bupivacaine alone. However, there has been no study to assess the effects of using sufentanil and midazolam in combination with bupivacaine.

Objectives: The aim of this study was to evaluate and compare properties (time of achievement/recovery of sensory/motor blocks; and time to request opium), complications (nausea, vomiting, shivering and hypotension), and neonatal first minute Apgar score with and without the addition of midazolam (M) or sufentanil (S) to bupivacaine (B) through intrathecal injection for spinal anesthesia, after the cesarean section.

Patients and Methods: In this double blind randomized clinical trial participants were randomly allocated to three equal groups: Group B (2.5 cc of bupivacaine $0.5 \%+1$ cc normal saline $0.9 \%$ ), Group BM (2.5 cc of bupivacaine $+0.02 \mathrm{mg} / \mathrm{kg}$ midazolam) and Group BS ( $2.5 \mathrm{cc}$ of bupivacaine $0.5 \%+0.7 \mathrm{cc}$ normal saline $0.9 \%+1.5 \mu \mathrm{g}$ of sufentanil, $0.3 \mathrm{cc}$ ). We used analysis of variance (ANOVA), post hoc test with Bonferroni adjustment, and chi-square test for statistical analysis; the analyses were performed using the SPSS-16 software. Given a significant level of 0.05 , overall and pair-wise comparisons were made.

Results: Seventy-five females participated in the study with no significant age difference (mean \pm standard deviation (SD): $28.60 \pm 6.06$, $28.12 \pm 5.29$ and $28.76 \pm 3.97$ year; $\mathrm{P}=0.9)$. Except for "time to motor block recovery" $(\mathrm{P}=0.057)$, the overall differences among the three groups was significant in terms of "time to sensory/motor block" $(\mathrm{P}<0.001)$, "time to sensory block recovery" $(\mathrm{P}<0.001)$, and "time to request opium" ( $\mathrm{P}<0.001)$. In all pair-wise comparisons there was no significant difference between the BM and BS group, except for "time to request opium", which was longer in the BS group $(\mathrm{P}<0.001)$. The occurrence of nausea $(\mathrm{P}=0.02)$, postoperative shivering $(\mathrm{P}=0.01)$ and hypotension $(\mathrm{P}<0.001)$ were significantly different between the groups, unlike vomiting, where the difference was not significant $(\mathrm{P}$ $=0.2$. . All neonates had an Apgar score of nine.

Conclusions: The findings showed that adding sufentanil or midazolam to bupivacaine shortens the onset of spinal anesthesia and increases the time duration of anesthesia; however it does not change the motor block recovery time. Adding sufentanil delays the first request for narcotic analgesics while adding midazolam leads to a decrease in nausea and hypotension. Adding sufentanil or midazolam does not have any deleterious effect on infants' Apgar scores. However, increases shivering in patients.
\end{abstract}

Keywords: Midazolam; Sufentanil; Bupivacaine; Analgesia; Cesarean Section

\section{Background}

The number of cesarean surgeries, especially in developed countries, has increased over the past two decades and this highlights the role of anesthesia in gynecologic and obstetric surgeries (1). Recently, there has been increasing tendency towards using regional anesthesia (RA) in cesarean section, which is associated with a reduction in the use of general anesthesia (GA). Because most pregnant women prefer to be awake and conscious during delivery and since RA may be considered as a safer method than GA, nowadays local blocks in cesarean delivery is used more commonly (2). Regional Anesthesia techniques have had great advances such as the reduction of the likelihood of errors; these techniques also lead to less bleeding than the surgery under GA. Although there are several different techniques for RA, spinal anesthesia is the easiest and the most economical compared to other methods. During spinal anesthesia there is a very low chance of pharmaceutical intoxication and the least amount of drug is transferred to the fetus.

There are many drugs for spinal anesthesia, each having its own advantages and disadvantages. The most commonly used drug for spinal anesthesia is bupivacaine, with a maximum effect time of 75 to 150 minutes. Although spinal anesthesia causes a proper height of analgesia up to the fourth thoracic vertebrae, patients may feel pain during surgery. During the postoperative peri-

Copyright (C) 2015, Iranian Society of Regional Anesthesia and Pain Medicine(ISRAPM). This is an open-access article distributed under the terms of the Creative Commons Attribution-NonCommercial 4.0 International License (http://creativecommons.org/licenses/by-nc/4.0/) which permits copy and redistribute the material just in noncommercial usages, provided the original work is properly cited. 
od, when the analgesic effect of local anesthetic drug is vanished, patients usually feel severe pain (3). Adjuvants in intrathecal analgesic injections and consumption of sedatives after the operation are associated with various risks and may be dangerous. Delayed effect and insufficient analgesic effect are among problems, which may occur when using bupivacaine alone for anesthesia $(3,4)$.

The addition of different drugs to reduce the adverse effects of anesthetic solution has been previously studied. There are benzodiazepine receptors throughout the nervous system, including the spinal cord, which show connections with gamma-aminobutyric acid (GABA) receptors (5). Midazolam is one of the drugs, which is categorized under the benzodiazepine group of drugs. This drug is used as a preoperative sedative or as a sleep medication during operations, and as a sedative drug during delivery, while it does not have analgesic effects (6); however, in some recent studies on intrathecal anesthesia, the drug has shown clear analgesic effects $(7,8)$. Various studies have found that adding midazolam to bupivacaine significantly increases the duration of postoperative analgesia (9). Sufentanil is another narcotic, which is a synthetic drug soluble in fat and is short-acting (10). Adding narcotics to spinal anesthetic drugs was first examined in 1979 through adding morphine to spinal anesthesia. Since morphine was soluble in water and had a long-acting effect, one of the major complications was delayed respiratory depression. Considering the fact that sufentanil is less soluble in water and has a short-acting effect while its effect starts quickly, it is a more useful and safer drug for spinal anesthesia $(11,12)$.

In this study, we conducted a clinical trial to examine the effect of adding midazolam and sufentanil to bupivacaine, through intrathecal injection for spinal anesthesia, on likely maternal and neonatal complications.

\section{Objectives}

The aim of the present study was to evaluate and compare various properties (time to achieve/recovery of sensory/motor blocks; and time to request opium), complications (nausea, vomiting, shivering and hypotension), and neonatal first minute Apgar with and without adding midazolam or sufentanil to bupivacaine, through intrathecal injection for spinal anesthesia after cesarean section.

\section{Patients and Methods}

This study was a double blind randomized controlled clinical Trial (RCT), registered with the code of IRCT2013052813488N1 in the Iranian registry of clinical trial. Prior to undertaking any action, the procedure of the project was approved by the ethics committee of Semnan University of Medical Sciences. The study population consisted of female volunteers for undergoing elective caesarean section admitted to Amir-Al-Momenin hospital of Semnan (Iran), during years 2012 and 2013. In this study we used the convenient sampling method, thus eligible mothers who were willing and consented to participate were enrolled in the study. In this study, the sample size was determined as 25 participants for each group regarding the occurrences of complications (nausea and vomiting) of spinal anesthesia in cesarean section according to the results of the study by Rasooli et al. (2009) (13); in which 44 preeclamptic patients were randomly assigned to two equal groups: bupivacaine + sufentanil $\left(\mathrm{P}_{1}=13 \%\right)$ and bupivacaine alone $\left(\mathrm{P}_{2}=54 \%\right)$; using two-sample comparison of proportions formula in which alpha $=0.05$ and power $=0.80$ :

$$
n=2 \frac{\left[(1-\bar{p}) \times \bar{p}\left(z_{1-\frac{\alpha}{2}}+z_{1-\beta}\right)^{2}\right]}{\left(p_{1}-p_{2}\right)^{2}}
$$

$$
p=\frac{p_{1}+p_{2}}{2}
$$

Eligible participants for this study included mothers who werfigure 1e categorized as group I and II in terms of American society of anesthesiologists (ASA) criteria, were aged between 18 to 40, had no prohibition for undergoing spinal anesthesia, were not allergic to topical anesthetics and the three drugs under the study, did not have a neuropathy, and consented to undergo spinal block. Patients were not included in the study: in case of the presence of gastrointestinal diseases, premature birth before 36 weeks, receiving anti-nausea medication during the 24 hours before surgery, having pregnancy induced hypertension or problems during cesarean section, and receiving narcotic drugs during the operation.

The patients were randomly divided to three groups using the random block method. For randomization we used the random block allocation method in which we had six blocks with three components: ABC, ACB, BAC, $\mathrm{BCA}, \mathrm{CAB}$ and $\mathrm{CBA}$. We applied the random digit number generator to select and sort the blocks. Using this method, the groups were either randomized or balanced.

The three groups included mothers who underwent spinal anesthesia with the following doses: Group B, 2.5 cc of bupivacaine 0.5\%+1 cc normal saline 0.9\%; Group BM, 2.5 cc of bupivacaine $+0.02 \mathrm{mg} / \mathrm{kg}$ midazolam; and Group BS: 2.5 cc of bupivacaine $0.5 \%+0.7$ cc normal saline $0.9 \%+$ $1.5 \mu \mathrm{g}$ of sufentanil $0.3 \mathrm{cc}$.

The patients were informed about the procedures and consented to participate before being enrolled in the study. After placing the patient on the operating table, an angiocath was installed on one hand of the patient and 100 cc of Ringer's solution was injected. Monitoring included electrocardiogram, pulse-oximetry, as well as noninvasive measurement of blood pressure and heart rate. Patients were told to sit, and after prepping the place with betadine, a spinal needle number 25 was used to inject one of the prepared solutions into the spinal cord between the third and fourth lumbar; the drug was injected slowly during five sec- 
onds. The solution used for each group contained: $2.5 \mathrm{cc}$ of bupivacaine $0.5 \%+1$ cc normal saline $0.9 \%$ for group B; 2.5 cC of bupivacaine $+0.02 \mathrm{mg} / \mathrm{kg}$ midazolam for group BM; and 2.5 cc of bupivacaine $0.5 \%+0.7 \mathrm{cc}$ normal saline $0.9 \%+1.5 \mu \mathrm{g}$ of sufentanil $0.3 \mathrm{cc}$ for group BS. After removing the spinal needle, patients were immediately placed in supine position and an oxygen mask with the flow of 6 liters per minute was set on the patient's face, while sensory block with a needle (test pin prick) measured the time required to reach analgesia level at T10 [sensory block onset]. In addition, the time of motor block was measured using the Modified Bromage scale $(1=$ complete block, 2 = almost complete block, 3 = partial block, $4=$ detectable weakness of hip flexion while in supine position, $5=$ no detectable weakness of hip flexion while supine, and $6=$ able to perform partial knee bend) (motor block onset). The criteria were evaluated every three minutes to achieve the point of interest. Injection, positions, and all the conditions were the same in all these three groups. Mean arterial pressure and heart rate were measured before and after the onset of analgesia, and after delivery, the infants' Apgar score at birth (at the end of the 1st minute) was calculated and recorded.

In the event of hypotension of less than $20 \%$ of the base, ephedrine was injected and if heart rate reached below 45 beats per minute atropine was injected. Shivering, nausea, vomiting and hypotension were recorded as well. After the surgery, the patient was transferred to the recovery unit and sensory block recovery time at T10 was recorded. The time when feet were able to move again was recorded and then the patient was transferred to the ward. The onset of pain was set when the patient required narcotic drugs and it was determined using a standard visual analog scale (VAS) in which the left end showed the no-pain point (0) and the right end indicated the worst imaginable pain point (10). When the patient requested analgesics or narcotics, she was asked to select a point between 0 - 10 with regards to her pain severity. When pain severity was four or less, only routine analgesics (diclofenac suppository, $100 \mathrm{mg}$ every eight hours) was administered, and when it was above four (based on VAS) in addition to the aforementioned drug, a narcotic (pethidine $25 \mathrm{mg}$ ) was administered for the patient.

The data obtained was analyzed using the SPSS software V.16. Quantitative data were presented as mean and standard deviation, while qualitative data were presented as frequencies. To compare qualitative and quantitative data between groups we used Analysis of Variance (ANOVA), chi-square test, and post hock test with Bonferroni adjustment. In this study, a significant level of 0.05 was used.

\section{Results}

In this study, 75 females who underwent cesarean section were studied. Each group consisted of 25 people. There was no patient with failed or partial block in our study. Table 1 presents the mean and standard deviations (SD) of participants' age as well as the evaluated properties of interest related to anesthesia (i.e. time to onset and time to recovery of sensory/motor blocks; and time to request opium) in the three groups. As presented in Table 1, there was no significant difference between the mean ages of the three groups.

The time required to achieve sensory block was $4.48 \pm$ 3.00 minutes in group B, $2.24 \pm 0.72$ minutes in group BM, and $3.08 \pm 1.15$ minutes in group BS; this difference was statistically significant $(\mathrm{P}<0.001)$. When comparing any of the three groups with each other in terms of the time to achieve sensory block, a significant difference was observed between group BM and group B $(\mathrm{P}<0.001)$ and group $\mathrm{B}$ and control group $\mathrm{BS}(\mathrm{P}=0.03)$; however, there was no significant difference between group BM and group $B S(P=0.269)$.

The time required to achieve motor block was $3.84 \pm 1.28$ minutes in group B, $1.80 \pm 0.76$ minutes in group BM, and $1.60 \pm 0.7$ minutes in group BS, with the difference being statistically significant $(\mathrm{P}<0.001)$. Differences between group BM and group $\mathrm{B}(\mathrm{P}<0.001)$ as well as between group BS and group B ( $\mathrm{P}<0.001)$ were significant, however there was no significant difference between groups $\mathrm{BM}$ and $\mathrm{BS}(\mathrm{P}=0.739)$.

The time required for sensory block recovery was 78.64 \pm 13.27 minutes in group B, $97.92 \pm 16.10$ minutes in group $\mathrm{BM}$, and $103.00 \pm 25.02$ minutes in group BS; this difference was statistically significant $(\mathrm{P}<0.001)$. Considering pair-wise comparison of sensory block recovery time, a significant difference was observed between groups $B M$ and $\mathrm{B}(\mathrm{P}=0.002)$ and groups $\mathrm{BS}$ and $\mathrm{B}(\mathrm{P}<0.001)$, however there was no significant difference between groups $\mathrm{BM}$ and $\mathrm{BS}(\mathrm{P}=0.608)$.

Motor block recovery time was $143.52 \pm 24.98$ minutes in group B, $146.32 \pm 20.67$ minutes in group BM, and $130.32 \pm$ 28.19 minutes in group BS; although the P value was notably small, the difference was not statistically significant

Table 1. Comparing the Variables of the Study in the Three Groups

\begin{tabular}{lcccc}
\hline Variable & Group B a,b & Group BM & Group BS & P Value \\
\hline Age, $\mathbf{y}$ & $28.60 \pm 6.06$ & $28.12 \pm 5.29$ & $28.76 \pm 3.97$ & 0.9 \\
Time to sensory block, min & $4.48 \pm 3.00$ & $2.24 \pm 0.72$ & $3.08 \pm 1.15$ & $<0.001$ \\
Time to motor block, min & $3.84 \pm 1.28$ & $1.80 \pm 0.76$ & $1.60 \pm 0.70$ & $<0.001$ \\
Time to sensory block recovery, min & $78.64 \pm 13.27$ & $97.92 \pm 16.10$ & $103.00 \pm 25.02$ & $<0.001$ \\
Time to motor block recovery, min & $143.52 \pm 24.98$ & $146.32 \pm 20.67$ & $130.32 \pm 28.19$ & 0.057 \\
Time to request opium, min & $117.28 \pm 48.69$ & $117.80 \pm 16.54$ & $192.20 \pm 39.63$ & $<0.001$ \\
\hline a & & & &
\end{tabular}

${ }^{\mathrm{a}}$ Data are presented as Mean \pm SD.

$\mathrm{b}$ For each group $(\mathrm{n}=25)$. 
Abdollahpour A et al.

\begin{tabular}{|c|c|c|c|c|}
\hline Complications & Group B $^{\text {a,b }}$ & Group BM & Group BS & P Value \\
\hline Nausea & $8(32)$ & $3(12)$ & $12(48)$ & 0.02 \\
\hline Vomiting & $5(20)$ & $1(4)$ & $4(16)$ & 0.2 \\
\hline Hypotension & $21(84)$ & $4(16)$ & $16(64)$ & $<0.001$ \\
\hline Postoperative shivering & $10(40)$ & $20(80)$ & $12(48)$ & 0.01 \\
\hline
\end{tabular}

$(\mathrm{P}=0.057)$. Pair-wise comparison of motor block recovery time showed no significant difference between groups. Although the difference between groups BM and SM was notable (146.32 vs.130.32), it was not significant $(P=0.065)$.

The time interval from anesthetic injection to the time when the patient requested analgesic drugs (opium) was $117.28 \pm 48.69$ minutes in group $\mathrm{B}, 117.80 \pm 16.54$ minutes in group BM, and 192.20 \pm 39.63 minutes in Group BS; the difference was statistically significant $(\mathrm{P}<0.001)$. Comparison of groups with each other in terms of the time when patients requested analgesic drugs revealed that there was no significant difference between the midazolam and control group $(\mathrm{P}=0.9)$, however there was a significant difference between the sufentanil group and the control group $(\mathrm{P}<0.001)$, and the midazolam group and the sufentanil group $(\mathrm{P}<0.001)$. Accordingly, those who received sufentanil requested analgesic drugs after a longer period of time.

Table 2 shows the frequencies of side-effects during the operation. The occurrence of nausea $(P=0.02)$, postoperative shivering $(\mathrm{P}=0.01)$ and hypotension $(\mathrm{P}<0.001)$ were significantly different between groups, yet this was not the case for vomiting $(\mathrm{P}=0.2)$. Nausea and hypotension were less frequent in group BM (12\% and 16\%, respectively), while shivering was more frequent (89\%) in the same group. Nausea was more frequent in group BS (48\%) while hypotension was more frequent in group B (84\%).

Finally, the infant's Apgar score at birth was similar in all three groups; all infants had first minute Apgar scores of nine at birth.

\section{Discussion}

The main objective of this study was to examine the effects of sufentanil or midazolam combined with bupivacaine on spinal anesthesia in elective cesarean. Considering intrathecal adjutants, previous studies have focused on adding sufentanil and/or midazolam to bupivacaine and compared each with the effect of using bupivacaine alone on anesthetic quality and properties.

In a study conducted by Karbasfrushan et al. it was shown that the combination of bupivacaine and intrathecal midazolam generated an effective anesthetic drug, which was used to reduce pain. Although the onset of sedation was faster, yet the incidence of nausea and vomiting was higher in the experimental group. The du- ration of effective analgesia and the time for regression of sensory analgesia was the same in both groups (14).

In another study conducted by Rasooli et al. the authors evaluated the effects of adding sufentanil to bupivacaine for spinal anesthesia in preeclamptic patients undergoing cesarean section. It was reported that bupivacaine in combination with sufentanil provided acceptable spinal anesthesia. The combination caused less hypotension and nearly eliminated the need for vasopressor support of blood pressure. In addition, it also decreased the incidence of nausea and vomiting, without negatively influencing neonatal outcome (13).

In a study conducted by Vyas et al. it was shown that adding sufentanil to bupivacaine significantly reduced the time required for spinal anesthesia. In addition, it was observed that the time required for motor block was significantly reduced among people receiving bupivacaine plus sufentanil (15). In a study by Sharifi et al.it was shown that adding midazolam to bupivacaine reduced the time required for spinal anesthesia. In that study it was shown that adding midazolam to bupivacaine reduced the time required for motor block. They also showed that adding midazolam to bupivacaine increased the time of spinal anesthesia (16). In another study conducted by Imani et al. it was found that adding midazolam to bupivacaine significantly reduced the time required for spinal anesthesia. It was also found that adding midazolam to bupivacaine reduced the time required for motor block (9). In a study conducted by Dahlgren et al. it was also shown that adding sufentanil to bupivacaine might increase the duration of anesthesia (17).

As noted in the aforesaid studies, adding sufentanil or midazolam to bupivacaine can commonly result in faster block in patients who undergo cesarean section. However, there are no previous studies that have compared the outcome of using sufentanil and midazolam in combination with bupivacaine. In our study no significant difference was observed between these two groups in terms of the onset of sensory and motor block. However in our study, sensory block recovery in the groups receiving sufentanil plus bupivacaine and midazolam plus bupivacaine took longer than in the control group, yet there was no difference between the two groups receiving the drug in combination with sufentanil and midazolam.

There was no significant difference between the three 
groups in terms of age, which indicates that the results are not influenced by age; this also proves effective randomization. The time required for spinal anesthesia onset in terms of time to sensory and motor block in the control group was significantly longer than that of the other groups, which received bupivacaine combined with sufentanil or midazolam; this shows the positive effect of adding these two drugs to bupivacaine. However, there was no difference between the group receiving bupivacaine and sufentanil (group BS) and the group receiving bupivacaine and midazolam (group BM).

According to the results of the present study, there was no significant difference between these three groups in terms of motor block recovery time; however, P was very small $(P=0.057)$ and this shows that an increase in the number of samples might lead to different results. There was no study available comparing these three groups in terms of motor block recovery time.

In this study there was no significant difference between the midazolam group and control group in terms of requesting analgesics by the patient; however, there was a significant difference between the sufentanil group and control group, and midazolam group and sufentanil group. This means that those who received sufentanil requested analgesics after a longer period of time. Thus, the findings of this study indicate that adding sufentanil to bupivacaine reduced pain after cesarean section, yet the addition of midazolam was not very effective. In a similar study, Lin et al. (1998) examined the effects of adding sufentanil to bupivacaine on spinal anesthesia for cesarean and reported positive effects with the addition of sufentanil to bupivacaine, which included in the reduction of the need for painkillers (18). In contrast to our results, in the study of Imani et al. it was found that adding midazolam to bupivacaine significantly led to a longer duration of time for the first request for analgesics (9). There was no study available that investigated the difference between the combination of sufentanil and bupivacaine, and midazolam and bupivacaine.

In the present study, adding midazolam to bupivacaine significantly reduced nausea in the patients. In this regard, adding midazolam was more effective than adding sufentanil to bupivacaine. The group receiving the combination of sufentanil plus bupivacaine did not significantly differ from the control group in terms of nausea. In a study conducted by Lee et al. in 2011, the results were similar to those of our study and it was shown that adding sufentanil to bupivacaine increased nausea (19). The results of the study by Imani et al. was also consistent with our results and it was found that adding midazolam to bupivacaine reduced nausea in the studied patients (9). Nevertheless, the study of Sharifi et al. had contradictory results (16). There is no study available investigating the difference between adding sufentanil to bupivacaine and midazolam to bupivacaine, simultaneously. In our study, no significant difference was found between the three groups in terms of the incidence of vomiting.
In this study, there was no significant difference in hypotension between the control group and the group receiving bupivacaine combined with sufentanil, yet adding midazolam to bupivacaine significantly decreased the rate of hypotension. In addition, comparing Group 2 (midazolam + bupivacaine) and Group 3 (sufentanil + bupivacaine), revealed that hypotension in Group 2 was significantly less than Group 3. Therefore, adding midazolam to bupivacaine was effective in reducing blood pressure yet adding sufentanil to bupivacaine had no effect. In the study of Vyas et al. it was found that people who received sufentanil plus bupivacaine did not significantly differ from the control group in terms of hypotension (15). In this study, adding midazolam to bupivacaine led to a significant increase in the level of shivering, which was higher than that in sufentanil and bupivacaine group and the control group. Accordingly, $80 \%$ of patients receiving midazolam had shivering $(40 \%$ of patients in the control group and $48 \%$ in the sufentanil group). However, there was no difference in shivering between the control group and the group receiving sufentanil. In this project, like other similar studies, we found that adding sufentanil or midazolam had no negative effect on neonatal Apgar score and in this study all infants had an Apgar score of nine at birth (16).

Overall, the findings showed that adding sufentanil or midazolam to bupivacaine shortened the onset of spinal anesthesia, and increased the time duration of anesthesia; however it did not change the motor block recovery time. Adding sufentanil delayed the first request for narcotic analgesics while adding midazolam led to a decrease in nausea and hypotension. Adding sufentanil or midazolam did not have any deleterious effects on the infants' Apgar. However, it increased shivering in patients.

\section{Acknowledgements}

This study was registered in the Iranian registry of clinical trial with registration code IRCT2013052813488N1. It was based on a dissertation conducted in 2014 in partial fulfillment of the requirements for certification of a general medical degree by Semnan University of Medical Sciences. We would like to sincerely appreciate all patients who spent their time and helped the researchers of this study. We also thank the staff of Semnan university of medical sciences for their supports in the implementation of this research project.

\section{Authors' Contributions}

Abolfazl Abdollahpour and Raheleh Azadi designed the study. Razieh Bandari prepared the manuscript and edited the final version. Majid Mirmohammadkhani performed the data analysis.

\section{Funding/Support}

This study supported by Semnan University of Medical Sciences, Semnan, Iran. 


\section{References}

1. Yeoh SB, Leong SB, Heng AS. Anaesthesia for lower-segment caesarean section: Changing perspectives. Indian J Anaesth. 2010;54(5):409-14.

2. Ghazi A, Raja Y. Combined low-dose spinal-epidural anaesthesia versus single-shot spinal anaesthesia for elective caesarean delivery. Int J Obstet Anesth. 2007;16(1):90-1.

3. Miller RD, Eriksson LI, Fleisher LA, Jeanine P, Kronish W, Young WL. Spinal,Epidural and caudal anesthesia, . Miller's Anesthesia. 7 ed. Philadelphia: Elsevier; 2010.

4. Tucker AP, Mezzatesta J, Nadeson R, Goodchild CS. Intrathecal midazolam II: combination with intrathecal fentanyl for labor pain. Anesth Analg. 2004;98(6):1521-7.

5. Olkkola KT, Ahonen J. Midazolam and other benzodiazepines. Handb Exp Pharmacol. 2008(182):335-60.

6. Goodchild CS, Guo Z, Musgreave A, Gent JP. Antinociception by intrathecal midazolam involves endogenous neurotransmitters acting at spinal cord delta opioid receptors. Br J Anaesth. 1996;77(6):758-63.

7. Yaksh TL, Allen JW. The use of intrathecal midazolam in humans: a case study of process. Anesth Analg. 2004;98(6):1536-45.

8. Dyer RA, Joubert IA. Low-dose spinal anaesthesia for caesarean section. Curr Opin Anaesthesiol. 2004;17(4):301-8.

9. Imani F, Mirdehghan MH, Entezary SR, Mehdizadeh Kashi A. Evaluation of Maternal and Neonatal Effects of Adding Midazolam to Bupivacaine under Combined Spinal-Epidural Anesthesia in Elective Cesarean Section. Razi J Med Sci. 2009;15(60):27-36

10. Miller RD, Eriksson LI, Fleisher LA, Jeanine P, Kronish W, Young WL. Pharmacokinetic Features of Individual Drugs. In: Miller RD, editor. Miller's Anesthesia. Philadelphia: Elsevier;2010. pp. 978-82.

11. Wang Q, She SZ, Zhang YF, Lao JX, Jin YL. [Effect of intrathecal administration of sufentanil at different doses on bupivacaine spinal anesthesia in gynecologic laparoscopy]. Nan Fang Yi Ke Da
Хие Хие Вао. 2008;28(8):1474-6.

12. Olofsson $C$, Nygards EB, Bjersten AB, Hessling A. Low-dose bupivacaine with sufentanil prevents hypotension after spinal anesthesia for hip repair in elderly patients. Acta Anaesthesiol Scand. 2004;48(10):1240-4.

13. Rasooli S, Parish M, Mahmoodpoor A, Moslemi F, Sanaie S. Effect of spinal low dose bupivacaine-sufentanyl for cesarean section in preeclamptic parturients on neonatal outcome. Shiraz E Med J. 2009;10(4):201-8.

14. Karbasfrushan A, Farhadi K, Amini-Saman J, Bazargan-Hejazi S, Ahmadi A. Effect of intrathecal midazolam in the severity of pain in cesarean section: a randomized controlled trail. Iran Red Crescent Med J. 2012;14(5):276-82.

15. Vyas N, Sahu DK, Parampill R. Comparative study of intrathecal sufentanil bupivacaine versus intrathecal bupivacaine in patients undergoing elective cesarean section. J Anaesthesiol Clin Pharmacol. 2010;26(4):488-92.

16. Sharifi SM, Sooki Z, Farhadi K, Karbasforushan A. Assessing the effect of intrathecal midazolam in the quality and duration of analgesia in cesarean section. Feyz J Kashan Univ Med Sci. 2007;11(1):8-12.

17. Dahlgren G, Hultstrand C, Jakobsson J, Norman M, Eriksson EW Martin H. Intrathecal sufentanil, fentanyl, or placebo added to bupivacaine for cesarean section. Anesth Analg. 1997;85(6):1288-93.

18. Lin BC, Lin PC, Lai YY, Huang SJ, Yeh FC. The maternal and fetal ef fects of the addition of sufentanil to $0.5 \%$ spinal bupivacaine for cesarean delivery. Acta Anaesthesiol Sin. 1998;36(3):143-8.

19. Lee JH, Chung KH, Lee JY, Chun DH, Yang HJ, Ko TK, et al. Comparison of fentanyl and sufentanil added to $0.5 \%$ hyperbaric bupivacaine for spinal anesthesia in patients undergoing cesarean section. Korean J Anesthesiol. 2011;60(2):103-8. 